\title{
Medial and Lateral Networks in Anterior Prefrontal Cortex Support Metacognitive Ability for Memory and Perception
}

\author{
Benjamin Baird, ${ }^{1}$ Jonathan Smallwood, ${ }^{2}$ Krzysztof J. Gorgolewski, ${ }^{3}$ and Daniel S. Margulies ${ }^{3}$ \\ ${ }^{1}$ Department of Psychological and Brain Sciences, University of California, Santa Barbara, California 93106, ${ }^{2}$ Department of Psychology, University of York, \\ North Yorkshire Y010 5DD, United Kingdom, and ${ }^{3}$ Max Planck Research Group: Neuroanatomy \& Connectivity, Max Planck Institute for Human Cognitive \\ and Brain Sciences, 04103, Leipzig, Germany
}

\begin{abstract}
Convergent evidence indicates that frontopolar Brodmann area 10, and more generally the anterior prefrontal cortex (aPFC), supports the human capacity to monitor and reflect on cognition and experience. An important unanswered question, however, is whether aPFC is a homogeneous region that supports a general-purpose metacognitive ability or whether there could be regional specialization within aPFC with respect to specific types of metacognitive processes. Previous studies suggest that the lateral and medial subdivisions within aPFC may support metacognitive judgments of moment-to-moment perceptual processes and assessments of information from memory stored over longer time scales, respectively. Here we directly compared intraindividual variability in metacognitive capacity for perceptual decisions and memorial judgments and used resting-state functional connectivity (rs-fcMRI) to relate this variability to the connectivity of the medial and lateral regions of aPFC. We found a behavioral dissociation in metacognitive ability for perceptual and memorial judgments. Furthermore, functional connectivity analysis revealed distinct patterns of connectivity that correlated with individual differences in each domain. Metacognitive ability for perceptual decisions was associated with greater connectivity between lateral regions of aPFC and right dorsal anterior cingulate cortex, bilateral putamen, right caudate, and thalamus, whereas metacognitive ability for memory retrieval predicted greater connectivity between medial aPFC and the right central precuneus and intraparietal sulcus/inferior parietal lobule. Together, these results suggest that an individual's capacity for accurate introspection in the domains of perception and memory is related to the functional integrity of unique neural networks anchored in the medial and lateral regions of the aPFC.
\end{abstract}

\section{Introduction}

Successful decision-making and action depend on accurately evaluating the success of basic cognitive processes that contribute to thought and behavior, a capacity known as "metacognition" (Metcalfe and Shimamura, 1994). Convergent evidence indicates that the frontopolar cytoarchitectonically defined Brodmann area 10 (BA 10), and more generally the anterior prefrontal cortex (aPFC), is a critical part of the neuroanatomical basis of metacognitive thought. Supporting a contribution to higher-level cognition, aPFC has shown substantial expansion in humans compared with nonhuman primates (Öngür et al., 2003). Furthermore, aPFC is the only prefrontal region that is almost exclusively connected to supramodal cortex, placing it at the nexus of an information processing hierarchy in which the outputs of lower-level operations may be integrated and evaluated (Christoff and Gabrieli, 2000; Ramnani and Owen, 2004). Consistent with a role of aPFC in metacognition, patients with traumatic injury to this region display a variety of

\footnotetext{
Received Feb. 20, 2013; revised Aug. 7, 2013; accepted Aug. 10, 2013.

Author contributions: B.B., J.S., and D.S.M. designed research; B.B. performed research; B.B., J.S., K.J.G., and D.S.M. analyzed data; B.B., J.S., K.J.G., and D.S.M. wrote the paper.

B.B. was supported by National Science Foundation Graduate Research Fellowship Grant DGE-0707430. We thank Stephen Fleming for helpful discussion and Philipp Haueis for assistance in conducting the research.

The authors declare no competing financial interests.

Correspondence should be addressed to Dr. Benjamin Baird, Department of Psychological and Brain Sciences, University of California, 552 University Road, Santa Barbara, CA 93106-9660. E-mail: baird@psych.ucsb.edu. DOI:10.1523/JNEUROSCI.0786-13.2013

Copyright $\odot 2013$ the authors $\quad 0270-6474 / 13 / 3316657-09 \$ 15.00 / 0$
}

metacognitive deficits, such as an inability to monitor disease symptoms or accurately appraise their own cognitive functioning (Belyi, 1987; Joseph, 1999). Furthermore, structural and functional variation in aPFC has been shown to predict variance in metacognitive ability within the healthy adult population (Fleming et al., 2010, 2012).

In recent years, neuroimaging and cytoarchitectonic studies have begun to establish the existence of functional subdivisions within aPFC, with a primary differentiation between medial and lateral regions (e.g., Gilbert et al., 2006). An important question is whether these subdivisions of aPFC play differential roles in specific types of metacognitive processes. One possibility is that metacognitive evaluations of memorial or perceptual information involve medial and lateral aPFC, respectively. An accumulating number of studies have linked lateral aPFC to metacognitive evaluations of dynamic perceptual processes (Fleming et al., 2010, 2012; Fleming and Dolan, 2012). In contrast, medial aPFC has been widely implicated in metacognitive assessments of memory retrieval, particularly for prospective "feeling-of-knowing" judgments (e.g., Schnyer et al., 2005), but also for retrospective confidence ratings (e.g., Moritz et al., 2006; Modirrousta and Fellows, 2008; Chua et al., 2009), and "reality monitoring" tasks (distinguishing whether a memory was internally or externally generated) (Simons et al., 2006, 2008). Furthermore, a recent study (McCurdy et al., 2013) found that gray matter volume in neuroanatomically distinct regions of the lateral aPFC and precuneus covaried with metacognitive ability for 
perception and memory, respectively. Together, these results raise the possibility that there could be distinct networks for metacognition of memory and perception.

Here we assessed the covariance between metacognitive ability in the domains of perception and memory to understand whether they reflect homogeneous or dissociable introspective capacities. We then used resting-state functional connectivity (rsfcMRI) (Fox et al., 2005; Seeley et al., 2007) to assess the relation between the functional architecture of aPFC and individual differences in metacognitive ability for perceptual decisions and memorial judgments. Based on previous studies, we evaluated whether metacognitive ability for memory and perception would be supported by differentiable functional networks associated with a medial-lateral subdivision of aPFC.

\section{Materials and Methods}

Participants. Sixty participants were recruited from the database of the Max Planck Institute for Human Cognitive and Brain Sciences and received standard remuneration for participation in the experiment. From this sample, eight participants were excluded from analysis: five participants had scanning parameters that did not match the others in the group and three participants were excluded because of excessive motion in resting state scans. The final sample consisted of 52 participants (21 males, age range 19-46 years, mean age 27 years). Signed informed consent was obtained from all participants before completing the study. All participants had normal or corrected to normal vision and had no history of neurological or psychiatric disease.

Stimuli. Stimuli and tasks were programmed in MATLAB version 7.9 (MathWorks) using the Psychophysics Toolbox version 3.0 (Brainard, 1997; Kleiner et al., 2007).

Stimuli for the perceptual decision task consisted of visual displays composed of six Gabor gratings arranged in a circle around a fixation point at an eccentricity of 7.0 visual degrees (see Fig. $1 A$ ). Each grating subtended 2.8 visual degrees and consisted of vertical alternating light and dark bars modulated at a spatial frequency of 2.2 cycles per visual degree at a contrast of $20 \%$. Stimuli were presented on a CRT monitor in a darkened room at a viewing distance of $\sim 60 \mathrm{~cm}$.

Stimuli for the memory retrieval task consisted of 290 neutral-valence noncomposite nouns selected from the CELEX database for German word norms (Baayen et al., 1995). All stimuli were 5 characters in length and had a word frequency between 6 and 150 per million.

Tasks and procedure. Participants performed two experimental sessions: a functional imaging session in which resting-state fMRI was acquired and a subsequent behavioral session in which they were asked to make metacognitive evaluations of perceptual and memorial decisions (for a schematic outline of the metacognitive tasks, see Figure 1). Task order was counterbalanced across participants.

The perceptual task was adapted from Fleming et al. (2010) and Song et al. (2011). Each trial (of 360 trials total, divided evenly into 4 blocks) consisted of a presentation of a $200 \mathrm{~ms}$ visual stimulus display consisting of six Gabor gratings arranged around central fixation, followed by a interstimulus interval (ISI) of $500 \mathrm{~ms}$ during which only the fixation cross remained on the screen, followed by a second $200 \mathrm{~ms}$ stimulus display consisting of 6 Gabors arranged around the fixation (see Fig. 1). In one of the two stimulus displays, the orientation of one of the Gabor patches was tilted slightly from the vertical axis. The display interval in which this "pop-out" Gabor occurred as well as its spatial location on the screen varied randomly across trials. The orientation of the pop-out Gabor was adjusted using a 2-up 1-down adaptive staircase procedure (Levitt, 1971; Fleming et al., 2010) designed to result in a convergence on $70 \%$ accuracy for individual performance. Two consecutive correct responses resulted in a reduction of the orientation parameter by one step, whereas one incorrect response resulted in an increase of the orientation parameter by one step. The orientation step size was 0.25 degree. After the offset of the second stimulus presentation, participants made unspeeded 2-choice discriminations as to whether the "pop-out" Gabor occurred in either the first or second stimulus display. Participants then rated their confidence in the accuracy of their response on a scale of 1 (low confidence) to 6 (high confidence) (Fleming et al., 2010). All responses were made using the number pad on the keyboard.

The memory task consisted of two phases: encoding and recognition. Before beginning the encoding phase, participants were informed that a recognition phase would follow in which their memory for the presented words would be tested. During encoding, participants viewed 145 words randomly selected from the full set of 290 word stimuli. Words were displayed sequentially in the center of the monitor for $1.5 \mathrm{~s}$ and were separated by an ISI of $1 \mathrm{~s}$ in which a fixation cross was displayed. During recognition, participants were presented with each word from the full list of stimuli in a random order (half of which were presented during encoding and half of which were new), and were asked to make unspeeded 2-choice discriminations as to whether the stimulus was old or new. Participants then rated their confidence in the accuracy of their response on a scale of 1 (low confidence) to 6 (high confidence) (Fleming et al., 2010). All responses were made using the number pad on the keyboard.

Quantification of metacognitive ability. Signal detection theory (SDT) (Green and Swets, 1966) was used to compute estimates of metacognitive accuracy, here quantified as the ability of an observer to discriminate between their own correct and incorrect perceptual decisions or memorial judgments with confidence ratings. A major concern in any metacognitive ("type II") analysis is to separate estimates of type II sensitivity from the potential confounding influences of type II response bias as well as sensitivity on the primary ("type I") task (e.g., Galvin et al., 2003). SDT offers a model-based approach that can account for these influences to quantify metacognitive accuracy independent of an observer's decision strategy or cognitive ability on the primary task.

Metacognitive accuracy on the perceptual task was quantified using the computational methods outlined in detail by Fleming et al. (2010). Because performance on the perceptual task is held constant with an online thresholding procedure, it is possible to compute a measure of metacognitive accuracy that is unconfounded by type I performance directly from the empirical type II receiver operating characteristic (ROC) curve. The type II ROC curve reflects the relationship between the accuracy of visual discriminations and the observer's confidence rating. For each level of confidence $i, p$ (confidence $=i \mid$ correct) and $p$ (confidence $=i \mid$ incorrect) were calculated, transformed into cumulative probabilities and used to construct each $x, y$ point on the empirical ROC curve (Galvin et al., 2003; Kornbrot, 2006; Fleming et al., 2010). The area under the type II ROC curve $\left(A_{\text {roc }}\right)$, when performance is held constant, provides a robust estimate of metacognitive discrimination that is independent of both type II response bias and type I sensitivity as follows: $A_{R O C}=0.25 \sum_{i=1}^{6}\left[\left(Y_{i+1}-X_{i}\right)^{2}-\left(Y_{i}-X_{i+1}\right)^{2}\right]+0.5$ (Kornbrot, 2006). Type I sensitivity $\left(d^{\prime}\right)$ was calculated as $d^{\prime}=z(H)-z(F A)$, where $z$ represents the inverse of the cumulative normal distribution and $H=p($ response $=1 \mid$ interval $=1)$ and $F A=p($ response $=1 \mid$ interval $=2$ )

Controlling for type I performance by experimental design in a recognition memory paradigm poses serious methodological challenges; therefore, quantification of metacognitive accuracy in the memory task required a computational approach that explicitly accounts for type I performance. A model-based SDT approach to account for variance in primary task performance in the computation of type II sensitivity has recently been described and validated (Maniscalco and Lau, 2012; McCurdy et al., 2013). This method has been discussed at length previously (Rounis et al., 2010; Maniscalco and Lau, 2012). Briefly, the approach exploits the link between type I and type II SDT models to express observed type II sensitivity at the level of the type I SDT model (termed meta $\left.d^{\prime}\right)$. Maximum likelihood estimation is used to determine the parameter values of the type I SDT model that provide the best fit to the observed type II data. A measure of metacognitive ability that controls for differences in type I sensitivity is then calculated by taking the ratio of meta $d^{\prime}$ and the type I sensitivity parameter $d^{\prime}: M_{\text {ratio }}=$ meta $d^{\prime} / d^{\prime}$. The most straightforward approach to computing $M_{\text {ratio }}$ involves an equal variance SDT model in which the variances of internal distributions of evidence for "old" and "new" in the type I model are assumed to be equal. However, this assumption may often be violated for 2-choice old/new recognition memory tasks (Swets, 1986; Mickes et al., 2007). We therefore also 
computed $M_{\text {ratio }}$ under an unequal variance SDT model. This approach uses the slope of the type I zROC to infer the ratio of the SDs of the type I distributions (s) underlying the two response categories (Macmillan and Creelman, 2004), and then holds this parameter constant in the estimation $M_{\text {ratio }}$. However, a significant limitation of this approach is that the estimation of $s$ is influenced by type II sensitivity, and estimates of $M_{\text {ratio }}$ under this approach may have reduced sensitivity because it controls for a parameter that is influenced by metacognitive accuracy. Type I sensitivity $\left(d^{\prime}\right)$ was calculated as $d^{\prime}=z(H)-z(F A)$, where $z$ represents the inverse of the cumulative normal distribution and $H=$ $p$ (response $=$ old $\mid$ stimulus $=$ old $)$ and $F A=p$ (response $=$ old|stimulus $=$ new)

MRI acquisition and analysis. Resting-state fMRI scans were collected on two scanners at the Max Planck Institute for Cognitive and Brain Sciences: a 3.0 Tesla Siemens Tim Trio scanner and a 3.0 Tesla Siemens Verio scanner. A T2*-weighted echo-planar imaging (EPI) sequence was used $\left(\mathrm{TR}=2000 \mathrm{~ms} ; \mathrm{TE}=30 \mathrm{~ms}\right.$; flip angle $=90^{\circ}$; acquisition matrix $=$ $64 \times 64 ; \mathrm{FOV}=192 \mathrm{~mm}$; acquisition voxel size $=3 \times 3 \times 4 \mathrm{~mm} ; 30$ interleaved slices). For reasons unrelated to the present investigation, there was some variability in the duration of the resting-state scans (number of volumes, 180-300). To control for any influence of scan duration or scanner model, we included these variables as nuisance covariates in all statistical analyses. During the resting-state scan, participants were instructed to relax, to hold as still as possible, and to keep their eyes open. Before the functional scan, high-resolution T1-weighted anatomical scans were also acquired (MPRAGE, TR $=2300 \mathrm{~ms}$; $\mathrm{TE}=2.96$ $\mathrm{ms}$; $\mathrm{TI}=900 \mathrm{~ms}$; flip angle $=9^{\circ} ; \mathrm{FOV}=256 \mathrm{~mm}$; acquisition voxel size $=1 \times 1 \times 1 \mathrm{~mm})$.

Structural (T1) data processing. Cortical surface reconstruction was performed on T1 scans using FreeSurfer (Dale et al., 1999; Fischl et al., 1999a, b, 2001, 2002, 2004a, b; Fischl and Dale, 2000; Ségonne et al., 2004; Han et al., 2006; Jovicich et al., 2006). For each subject, nonlinear transformation from $\mathrm{T} 1$ to the $2 \mathrm{~mm}$ MNI 152 template was calculated using ANTs (Avants et al., 2011).

Resting state $F M R I$ (EPI) data processing. Preprocessing was performed with a workflow from Brain Imaging Pipelines (https://github. com/INCF/BrainImagingPipelines) and all data processing integrated using Nipype (Gorgolewski et al., 2011). Modified preprocessing scripts may be found at https://github.com/NeuroanatomyAndConnectivity/ pipelines. The following specifications were used: To remove potential scanner instability effects, the first four volumes of each EPI sequence were removed. This was followed by simultaneous slice timing and motion correction using 4DRealign implemented in nipy (http://nipy. org/nipy) (Roche, 2011). Affine transformation from mean EPI image to T1 volume was calculated using BBRegister (Greve and Fischl, 2009). Brain mask, CSF mask, and white matter (WM) mask were extracted from FreeSurfer parcellation and transformed into EPI space (thresholded at 0.5 after interpolation). Realigned time series were masked using the brain mask. Principal components of physiological noise were estimated using CompCor (Behzadi et al., 2007). The joined WM and CSF masks and voxels of highest variance were used to extract two sets of principal components (also known as aCompCor and tCompCor). Outliers in the EPI sequence were discovered based on intensity and motion parameters (ArtDetect; http://www.nitrc.org/projects/artifact_detect). This was followed by denoising of the time series using a GLM model with motion parameters, CompCorr components, and outliers as regressors (note that global signal was not regressed). Time series were also smoothed using SUSAN with $5 \mathrm{~mm}$ full width half minimum kernel (Smith, 1992). Finally, high-pass $(0.01 \mathrm{~Hz})$ and low-pass $(0.1 \mathrm{~Hz})$ filters were applied using FSL. Quality of scans and preprocessing was assessed visually by looking at EPI to T1 coregistration overlay, motion parameter plots, and temporal signal-to-noise ratio volumes. Because of substandard data quality (too high motion or signal dropouts), three participants were discarded from further analysis.

To estimate connectivity, two spherical ROIs of $6 \mathrm{~mm}$ diameter with centers at 6, 58, 0 (medial aPFC) and 24, 58, 18 (lateral aPFC) were defined in the MNI152 space (see Fig. 4A). The location of the lateral aPFC seed region was based on the region described by Fleming et al. (2010). Our central coordinate was derived by centering the spherical
ROI on the peak voxel reported in Fleming et al. (2010) and then shifting it posteriorly in the coronal axis to ensure that the sphere was securely constrained within the pial surface of the cortex and remained within the morphological feature of the Fleming et al. (2010) coordinate. The medial aPFC seed region was based on the region described in a metaanalysis (Gilbert et al., 2006), which partitioned aPFC according to a classification algorithm. Because no strict stereotaxic definition of medial aPFC was supplied, we derived our selection of related coordinates by matching the prefrontal sulci of our template to the results of the classification partition $(z=0)$, constraining the circumference of the seed region to within a single hemisphere's gray matter $(x=6)$, and matching both medial and lateral seed regions in the coronal plane $(y=58)$. Although the two regions were not precisely identical in stereotaxic space to the activation peaks reported by Fleming et al. (2010) and Gilbert et al. (2006), they were the only regions examined within these areas of the cortex, and were selected to accommodate the constraints of the cortical structure while still assessing the same cortical regions described in the respective studies. ROI masks were transformed back to each subject EPI space using combined inverse nonlinear MNI-152-to-T1 transform and affine T1-to-EPI (thresholded at 0.5 after interpolation). Translated ROIs were restricted with the brain mask. ROI time series were estimated by averaging voxels within each ROI. Full brain connectivity (correlation) maps were calculated using AFNI (Cox, 2012). Connectivity maps were $z$-transformed using Fisher's $r$-to- $z$ transform and then spatially transformed into MNI152 space.

Statistical analyses. Group-level analysis was conducted using the GLM framework implemented in SPM8 (Wellcome Trust Department of Imaging Neuroscience, University College London). For each individual seed, voxelwise multiple regression analyses were performed with the connectivity maps, metacognitive accuracy scores, and nuisance covariates for age and gender. Additionally, to control for scan length and scanner model (see MRI acquisition and analysis) these variables were entered as simultaneous nuisance regressors. In addition to evaluating each seed point individually, we examined how the differential connectivity between medial and lateral aPFC related to memorial and perceptual metacognitive accuracy. For each individual, whole-brain connectivity maps of medial and lateral aPFC were subtracted from each other and voxelwise multiple regression was performed on the difference maps. Whole-brain analyses were conducted, correcting for multiple comparisons using topological FDR (Chumbley et al., 2010). Cluster forming threshold was set at $p<0.005$, and cluster size threshold was set at $p<0.05$ (FDR corrected). Unthresholded statistical maps were uploaded to NeuroVault.org database and are available at http://neurovault.org/collections/17/.

\section{Results}

\section{Behavioral performance}

In a counterbalanced design, participants $(N=52)$ completed a perceptual discrimination task and verbal recognition memory task in which they made 2-choice discriminations and then rated their confidence in the accuracy of their responses on a trial-by-trial basis (Fig. 1) (Fleming et al., 2010; McCurdy et al., 2013). The perceptual task was performed at an individually determined threshold using a 2-up 1-down adaptive staircase procedure that results in a convergence on $70 \%$ accuracy at the limit for individual performance (Levitt, 1971; Fleming et al., 2010). Analysis revealed that performance accuracy was well controlled by the staircase for all participants (mean 0.70 , SD 0.01 , range $0.66-$ $0.72)$. Overall, performance on the memory task was good and had similar mean accuracy (mean 0.71 , SD 0.09 , range $0.57-$ $0.91)$.

A linear mixed model with participant included as a random effect revealed that RT significantly predicted confidence at the trial level in both the perceptual decision task $(t=-48.03, p<$ 0.001 ; int $=3.48$, estimate $=-0.50)$ and memory retrieval task $(t=-22.51, p<0.001$; int $=4.14$, estimate $=-0.09)$, indicating that more confident decisions were associated with faster responses. Overall, mean confidence was higher in the memory retrieval task (mean 3.95, SD 0.77) compared with the perceptual 
A Perceptual Decision Task: Schematic of Single Trial $(\mathrm{N}=360)$

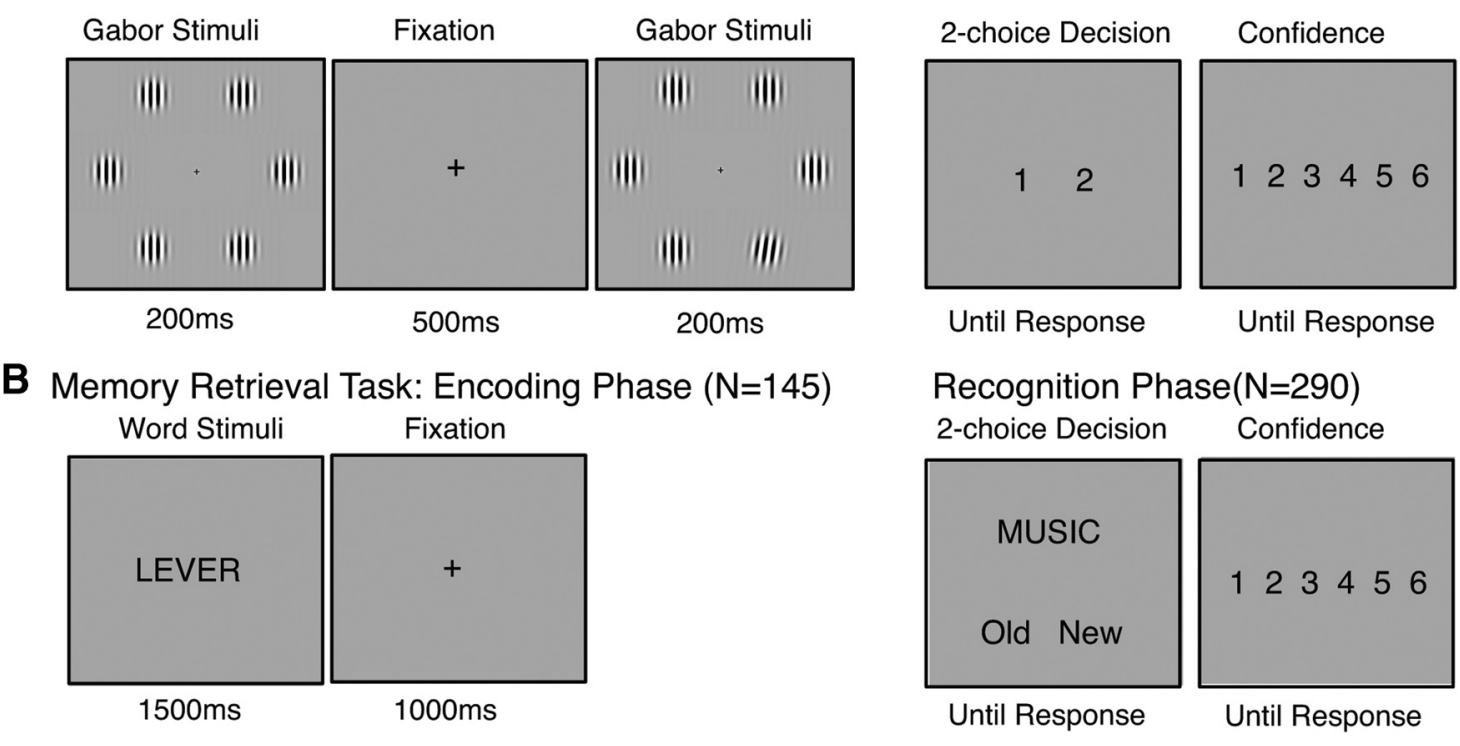

Figure 1. Experimental paradigm. Participants completed 2 tasks in a counterbalanced order. $A$, Perceptual discrimination task. Each trial $(N=360)$ consisted of a visual display of 6 Gabor gratings, followed by an ISI of $500 \mathrm{~ms}$, followed by a second visual display of 6 Gabor gratings. In one of the two displays, the orientation of one randomly selected Gabor patch was tilted slightly from the vertical axis. The orientation angle of this pop-out Gabor was adjusted using a 2-up 1-down adaptive staircase procedure. Participants made unspeeded 2-choice discrimination judgments as to whether the "pop-out" Gabor occurred in either the first or second stimulus display, then rated their confidence in the accuracy of their response on a scale of 1 (low confidence) to 6 (high confidence). $B$, Memory retrieval task. The memory task consisted of a classic verbal recognition memory paradigm. During encoding, participants viewed 145 words randomly selected from a set of 290 words. During recognition, participants were presented with each word from the full list of stimuli in a random order (half of which were presented during encoding and half of which were new) and were asked to make unspeeded 2-choice discrimination judgments as to whether the stimulus was old or new, and then rated their confidence in their response.

decision task (mean 2.80, SD 0.87) $\left[t_{(51)}=9.74, p<0.001\right]$, which may be attributed to the relative difficulty of the perceptual task that was performed at an individually determined perceptual threshold. Mean confidence level also showed a significant correlation within individuals across the two tasks $(r(50)=0.49, p<$ 0.001 ). Together, these results replicate previous findings (Song et al., 2011; Fleming et al., 2012) and suggest that confidence level reflects both a task-independent general level of confidence particular to an individual as well as a task-dependent level of confidence an individual has toward performance on a particular cognitive task.

SDT (Green and Swets, 1966) was used to quantify individual differences in metacognitive ability ("type II sensitivity"), here quantified as the ability to accurately link confidence with performance. SDT enables a model-based approach to the computation of type II sensitivity that is independent of response bias and type I sensitivity $\left(d^{\prime}\right)$ on the primary task. Analysis confirmed that metacognitive ability in both the perceptual decision task $\left(A_{\text {roc }}\right)$ and recognition memory task $\left(M_{\text {ratio }}\right)$ were uncorrelated with type I performance $\left(A_{\text {roc }}: r(50)=0.09, p=0.51 ; M_{\text {ratio }}\right.$ : $r(50)=0.16, p=0.22)$, or type II response bias $\left(A_{\text {roc }}: r(50)=\right.$ $-0.03, p=0.86 ; M_{\text {ratio }}: r(50)=0.08, p=0.57$ ). Additionally, orientation discrimination threshold in the perceptual task was uncorrelated with perceptual $A_{\text {roc }}(r(50)=-0.02, p=0.91)$, indicating that $A_{\text {roc }}$ estimates were not confounded with variance in perceptual acuity. SDT estimates of metacognitive ability were thus confirmed to be independent of response bias and variance in primary task performance, allowing a direct comparison of metacognitive ability across process domains. Analysis revealed that metacognitive accuracy for perceptual decisions $\left(A_{\text {roc }}\right)$ and memorial judgments $\left(M_{\text {ratio }}\right)$ were uncorrelated across individuals $(r(50)=0.03, p=0.81)$, indicating an intraindividual dissociation in metacognitive ability across process domains (Fig. 2). To ensure that this result was not an artifact of the fact that

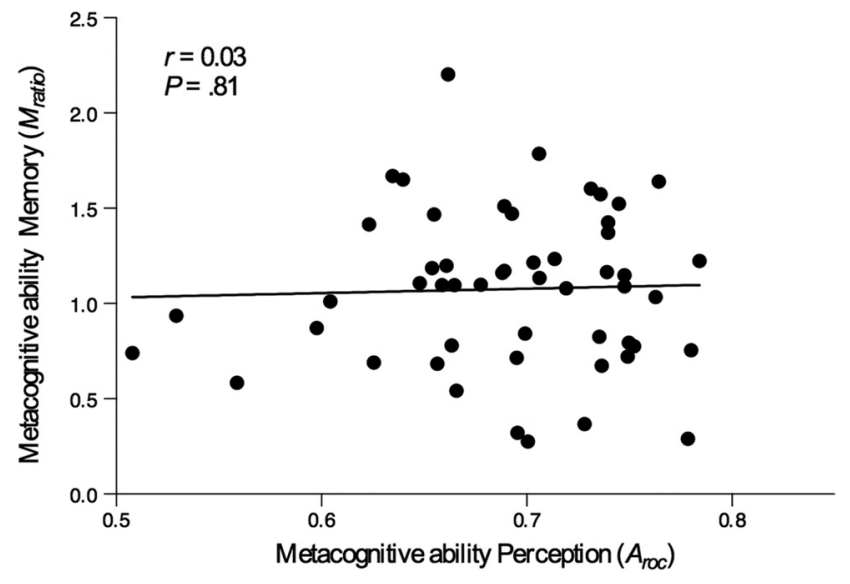

Figure 2. Behavioral results. Scatterplot of zero-order correlation between metacognitive accuracy for perceptual decisions $\left(A_{\text {roc }}\right)$ and memorial judgments $\left(M_{\text {ratio }}\right)[r(50)=$ $0.03, p=0.81]$.

metacognitive ability for memory and perception were in different units $\left(M_{\text {ratio }}\right.$ and $\left.A_{\text {roc }}\right)$, we calculated $M_{\text {ratio }}$ for the perceptual discrimination task and correlated it with $M_{\text {ratio }}$ for the memory task. These measures were also uncorrelated across individuals $(r(50)=-0.13, p=0.35)$, indicating that the lack of correlation between perceptual and memorial metacognitive ability in our data cannot be attributed to differences in the scale between $M_{\text {ratio }}$ and $A_{\text {roc }}$.

Functional connectivity of medial and lateral seed regions Seed regions were chosen for intrinsic functional connectivity analysis based on literature that localized medial and lateral aPFC subregions. Two spherical ROIs of $6 \mathrm{~mm}$ diameter with centers at 6, 58, 0 (medial aPFC; reflecting the area described by Gilbert et 


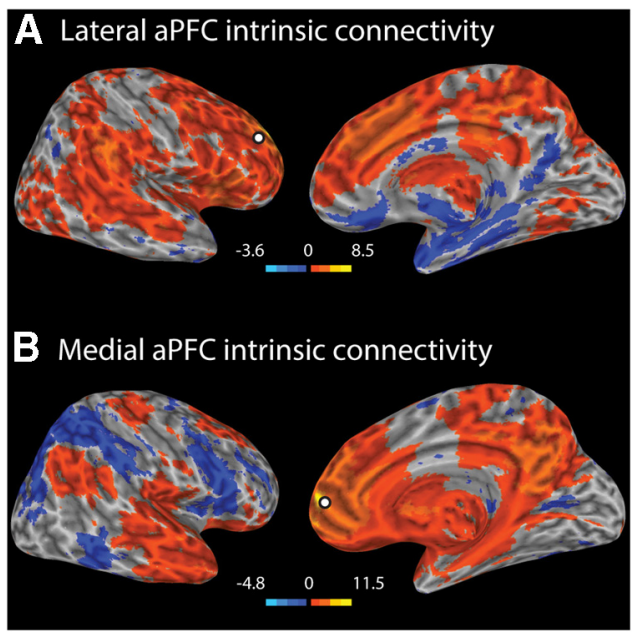

Figure 3. $\quad \boldsymbol{A}$, Lateral aPFC connectivity. Right lateral aPFC showed intrinsic connectivity with a broad network, including bilateral regions of superior frontal gyrus, cingulate gyrus, inferior frontal gyrus, precuneus, postcentral gyrus, inferior parietal lobule, lateral temporal cortex, orbital frontal cortex, thalamus, basal ganglia, caudate, insula, and cerebellum. $B$, Medial aPFC connectivity. Right medial aPFC displayed connectivity with bilateral regions of medial prefrontal cortex, orbital frontal cortex, precuneus, inferior parietal lobule, lateral temporal cortex, precentral gyrus, posterior and anterior cingulate, hippocampal formation, insula, thalamus, inferior occipital gyrus, and cerebellum.

al., 2006; meta-analysis) and 24, 58, 18 (lateral aPFC; reflecting the area described by Fleming et al., 2010; see Resting state fMRI (EPI) data processing) were defined in MNI152 space. Based on previous studies showing right aPFC lateralization to metacognitive processes (Schnyer et al., 2004; Schmitz et al., 2006; Fleming et al., 2010, 2012; Yokoyama et al., 2010), our seed regions were defined in medial and lateral divisions of right aPFC (see Fig. 4A). Right lateral aPFC showed intrinsic connectivity with a broad network, including bilateral regions of superior frontal gyrus, cingulate gyrus, inferior frontal gyrus, precuneus, postcentral gyrus, inferior parietal lobule, lateral temporal cortex, orbital frontal cortex, thalamus, basal ganglia, caudate, insula, and cerebellum (Fig. 3A). Right medial aPFC displayed connectivity with bilateral regions of medial prefrontal cortex, orbital frontal cortex, precuneus, inferior parietal lobule, lateral temporal cortex, precentral gyrus, posterior and anterior cingulate, hippocampal formation, insula, thalamus, inferior occipital gyrus, and cerebellum (Fig. $3 B$ ).

\section{Functional connectivity and metacognitive ability}

As shown in Figure $4 B$ (top two panels) and Table 1, metacognitive accuracy for perceptual decisions $\left(A_{\text {roc }}\right)$ was significantly correlated with functional connectivity between the right lateral aPFC seed region and three clusters: right dorsal anterior cingulate cortex (dACC), left putamen, and a cluster, including right putamen, right caudate, and thalamus. Metacognitive accuracy for memory retrieval $\left(M_{\text {ratio }}\right)$ did not significantly correlate with functional connectivity from the lateral aPFC seed region.

By contrast, metacognitive accuracy for perceptual decisions $\left(A_{\text {roc }}\right)$ did not correlate with functional connectivity with the medial aPFC seed region. As shown in Figure $4 B$ (bottom two panels) and Table 1, metacognitive accuracy on the memory retrieval task $\left(M_{\text {ratio }}\right)$ was significantly correlated with functional connectivity between the right medial aPFC seed region and two clusters in the right parietal cortex: central precuneus and intraparietal sulcus (IPS)/inferior parietal lobule (IPL). We conducted a follow-up analysis in which $M_{\text {ratio }}$ was estimated with an unequal variance SDT model ( $M_{\text {ratio }}$ [uneq var]; see Quantification of metacognitive ability). We found that $M_{\text {ratio }}$ [uneq var] also significantly predicted connectivity between medial $\mathrm{aPFC}$ and right central precuneus and right IPL/IPS (height threshold $p<0.005$; cluster corrected, $p<0.001 ; 1080$ voxels; peak voxel (MNI): $32,-46,34)$, as well as a cluster in right middle frontal gyrus (height threshold $p<$ 0.005; cluster corrected, $p=0.001 ; 445$ voxels).

Finally, we conducted a whole-brain analysis examining how the differential connectivity between medial and lateral aPFC related to memorial and perceptual metacognitive accuracy. We subtracted whole-brain connectivity maps between medial and lateral aPFC for each individual and performed voxelwise multiple regression analyses on the difference maps. We found six clusters where memorial metacognitive accuracy modulated the relative connectivity with our medial and lateral aPFC seed regions. These regions included the bilateral parahippocampal gyrus, precentral gyrus, precuneus, fusiform gyrus, and lingual gyrus (Fig. 5; Table 2). Higher metacognitive accuracy for memory was associated with a relative increase in the coupling of these regions with medial aPFC compared with connectivity with lateral aPFC. No significant regions were found where connectivity with medial aPFC correlated more highly with perceptual metacognitive accuracy compared to connectivity with lateral aPFC. In the reverse contrast (lateral aPFC-medial aPFC), no regions significantly correlated with either perceptual metacognitive accuracy or memorial metacognitive accuracy.

\section{Discussion}

We found that the capacity of an individual to make metacognitive evaluations of perceptual decisions and the ability to evaluate the contents of memory were independent from one another, indicating that metacognitive skill in one domain may not necessarily translate to another. Furthermore, metacognitive accuracy in each domain was related to distinct patterns of functional connectivity involving the medial and lateral regions of aPFC. Metacognitive ability for perceptual decisions was associated with increased connectivity between lateral aPFC and a network of regions involving right dACC, bilateral putamen, right caudate, and thalamus. In contrast, the capacity for accurate metacognitive assessments of memory was related to increased connectivity between medial aPFC and a network of regions, including the right central precuneus and IPS, spreading to the adjacent IPL. An analysis of the differential connectivity between medial and lateral seeds revealed that regions, including the hippocampal formation, precuneus, fusiform gyrus, lingual gyrus, and precentral gyrus, showed a relative increase in connectivity with medial compared with lateral aPFC with greater memorial metacognitive accuracy. Together, these results suggest that metacognitive accuracy in the domains of perception and memory is related to the functional integrity of unique neural networks anchored in the medial and lateral regions of the aPFC.

Our finding that metacognitive accuracy for perceptual discriminations was linked to connectivity with lateral aPFC is consistent with the finding that gray matter volume in this area correlates with the ability to link confidence with accurate decision-making for perceptual decisions (Fleming et al., 2010; McCurdy et al., 2013). Furthermore, the finding that metacognitive ability for perceptual decisions was related to connectivity between right lateral aPFC and AACC is consistent with the finding that these regions show increased activation during metacognitive assessments of visual discriminations and that the strength 
A

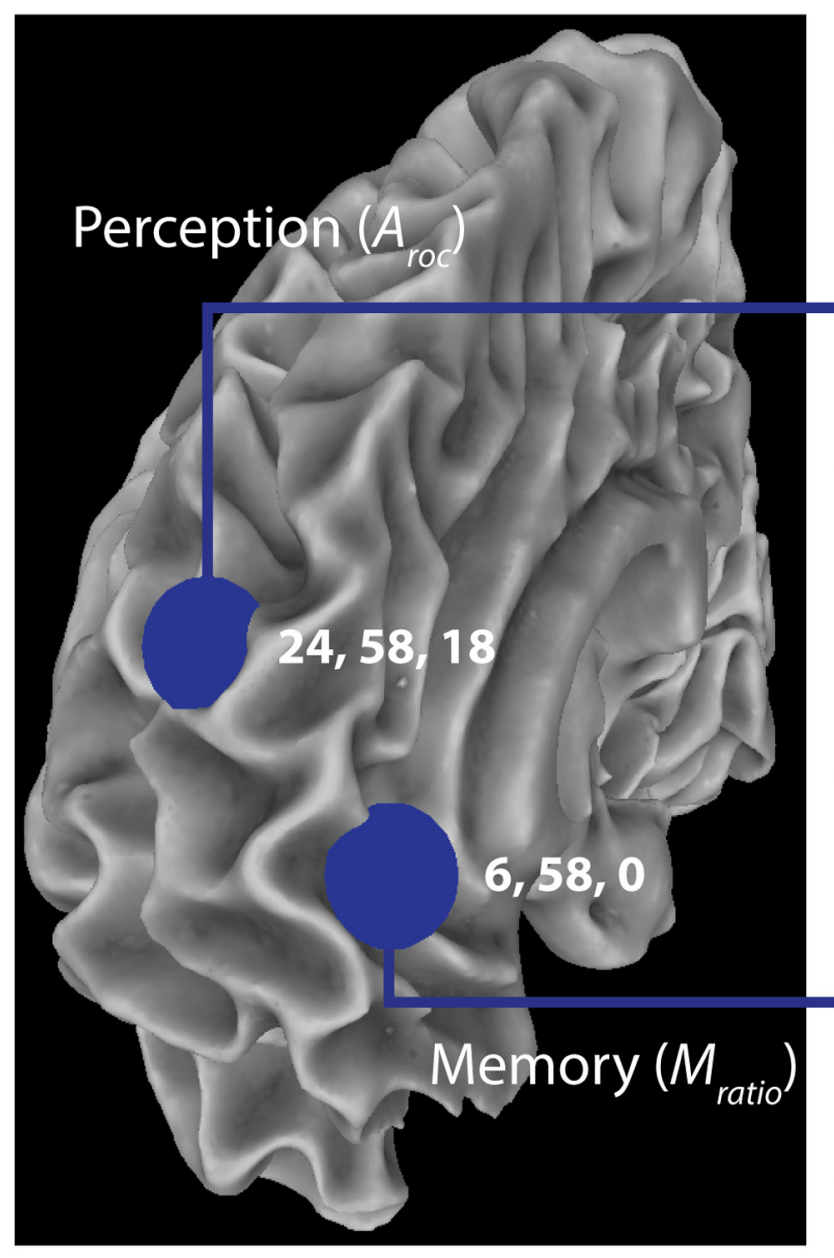

B
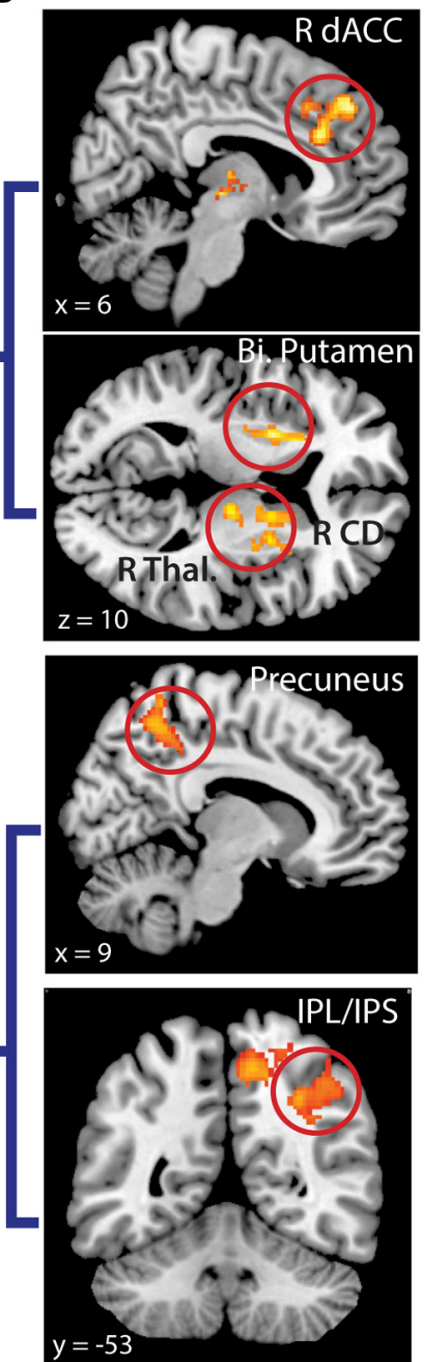

C

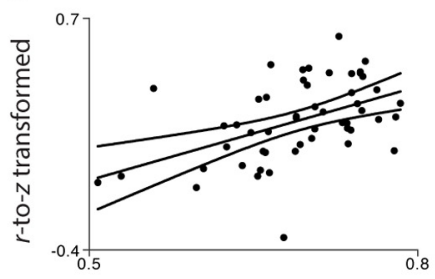

Metacognitive ability Perception

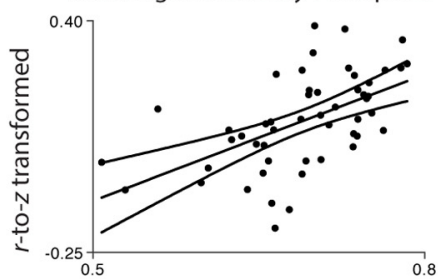

Metacognitive ability Perception

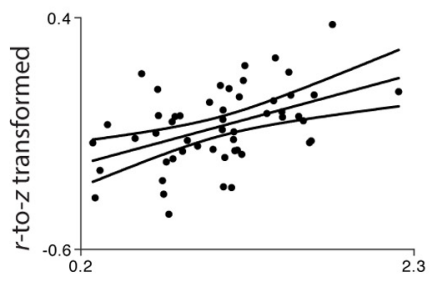

Metacognitive ability Memory

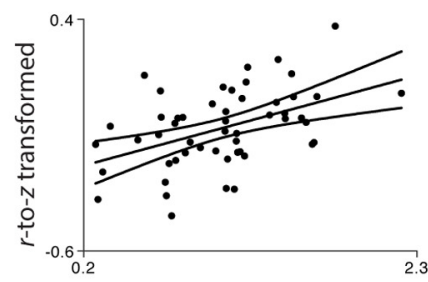

Metacognitive ability Memory

Figure 4. A, Seed regions. To estimate connectivity, two spherical ROls of $6 \mathrm{~mm}$ diameter with centers at 6,58, 0 (medial aPFC; reflecting the area described by Gilbert et al., 2006; meta-analysis) and 24, 58, 18 (lateral aPFC; reflecting the area reported by Fleming et al. (2010)) were defined in MNI152 space. B, fMRI connectivity results. Top two panels, Metacognitive accuracy for perceptual decisions is associated with increased connectivity between lateral aPFC seed region and right $\mathrm{AACC}$, bilateral putamen, right caudate, and thalamus. Bottom two panels, Metacognitive accuracy for memory is associated with increased connectivity between medial aPFC and right precuneus and right IPS/IPL. All clusters are significant at $p<0.05$, corrected for multiple comparisons using topological FDR (cluster forming threshold, $p<0.005$ ). C, Correlation between metacognitive accuracy scores and mean normalized correlation values of significant clusters.

Table 1. Functional connectivity of medial and lateral aPFC correlated with metacognitive accuracy for memory and perception ${ }^{a}$

\begin{tabular}{|c|c|c|c|c|c|c|}
\hline \multirow[b]{2}{*}{ Region } & \multirow{2}{*}{$\begin{array}{l}\text { Volume } \\
\left(\mathrm{mm}^{3}\right)\end{array}$} & \multirow{2}{*}{$\begin{array}{l}\text { Peak } \\
z \text {-value }\end{array}$} & \multirow{2}{*}{$\begin{array}{l}p \text { (cluster } \\
\text { FDRc) }\end{array}$} & \multicolumn{3}{|c|}{ Peak MNI } \\
\hline & & & & $x$ & $y$ & $z$ \\
\hline \multicolumn{7}{|l|}{ Memory $\left(M_{\text {ratio }}\right)$} \\
\hline Right IPL/IPS & 542 & 4.91 & 0.001 & 30 & -48 & 36 \\
\hline Right precuneus & 488 & 3.93 & 0.001 & 8 & -48 & 52 \\
\hline \multicolumn{7}{|l|}{$\begin{array}{c}\text { Perception }\left(A_{\text {rod }}\right) \\
\text { Lateral aPFC }\end{array}$} \\
\hline Right dACC & 390 & 3.87 & 0.001 & 8 & 42 & 42 \\
\hline $\begin{array}{l}\text { Right putamen, right caudate, } \\
\text { thalamus }\end{array}$ & 602 & 3.95 & $>0.001$ & 12 & -12 & 6 \\
\hline Left putamen & 245 & 4.05 & 0.014 & -24 & 14 & 8 \\
\hline
\end{tabular}

of activation in these regions during metacognitive judgments correlates with reported confidence (Fleming et al., 2012).

In contrast, the ability to make accurate metacognitive assessments of memory was associated with increased connectivity be- tween medial aPFC, the right central precuneus, and IPL/IPS, a pattern that is supported by previous literature. Functional data suggest that monitoring the contents of memory is associated with increased activity in medial prefrontal and lateral parietal regions (Chua et al., 2006, 2009). IPL also shows greater activation for high confidence hits in meta-memory tasks (Wheeler and Buckner, 2004; Kim and Cabeza, 2007) as well as strong "feelingof-knowing" judgments for semantic and episodic information (Elman et al., 2012). Furthermore, patients with parietal lesions produce fewer high confidence recognition responses during retrieval (Davidson et al., 2008; Simons et al., 2010) and lesions to the medial prefrontal cortex lead to impairments in evaluating memory (Pannu and Kaszniak, 2005; Modirrousta and Fellows, 2008). Finally, a recent voxel-based morphometry study by McCurdy et al. (2013) found that gray matter volume in the precuneus correlated with better metacognitive ability for memory.

Together our findings suggest that the accuracy with which an individual is able to evaluate lower-level processes in part depends on the domain of the metacognitive judgment. Metacog- 

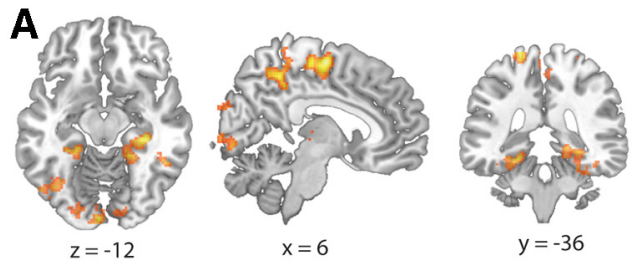

B

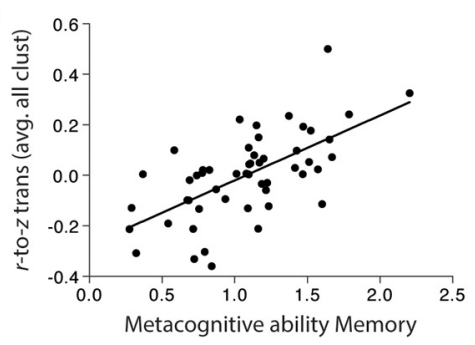

Figure 5. Differential functional connectivity between medial and lateral aPFC correlated with metacognitive accuracy for memory. $\boldsymbol{A}$, Medial aPFC-lateral aPFC connectivity correlated with metacognitive accuracy for memory predicted 5 clusters, including the hippocampal formation, precuneus, fusiform gyrus, lingual gyrus, and precentral gyrus (Table 2). All clusters are significant at $p<0.05$, corrected for multiple comparisons using topological FDR (cluster forming threshold, $p<0.005$ ). $\boldsymbol{B}$, Correlation between metacognitive accuracy scores and mean normalized correlation values of medial-lateral aPFC functional connectivity of all significant clusters.

Table 2. Differential functional connectivity between medial and lateral aPFC correlated with metacognitive accuracy for memory ${ }^{a}$

\begin{tabular}{|c|c|c|c|c|c|c|}
\hline \multirow[b]{2}{*}{ Region } & \multirow{2}{*}{$\begin{array}{l}\text { Volume } \\
\left(\mathrm{mm}^{3}\right)\end{array}$} & \multirow{2}{*}{$\begin{array}{l}\text { Peak } \\
z \text {-value }\end{array}$} & \multirow{2}{*}{$\begin{array}{l}p \text { (cluster } \\
\text { FDRc) }\end{array}$} & \multicolumn{3}{|c|}{ Peak MNI } \\
\hline & & & & $x$ & $y$ & $z$ \\
\hline $\begin{array}{l}\text { Right parahippocampal gyrus, } \\
\text { right fusiform gyrus }\end{array}$ & 913 & 4.96 & $<0.001$ & 34 & -28 & -16 \\
\hline $\begin{array}{l}\text { Precentral/postcentral gyrus, } \\
\text { MFG, precuneus }\end{array}$ & 3445 & 4.69 & $<0.001$ & 18 & -22 & 68 \\
\hline Right lingual gyrus, right MOG & 1621 & 4.18 & $<0.001$ & 28 & -86 & 14 \\
\hline Left fusiform gyrus, left MOG & 367 & 4.10 & 0.003 & -46 & -72 & -8 \\
\hline $\begin{array}{l}\text { Left parahippocampal gyrus, } \\
\text { left fusiform gyrus, left } \\
\text { lingual gyrus }\end{array}$ & 272 & 3.89 & 0.012 & -26 & -38 & -16 \\
\hline $\begin{array}{l}\text { Left insula, left precentral } \\
\text { gyrus }\end{array}$ & 296 & 3.55 & 0.009 & -46 & -12 & 22 \\
\hline
\end{tabular}

${ }^{a}$ All clusters significant at $p<0.05$, FDR corrected (height threshold, $p<0.005$ ).

MFG, Medial frontal gyrus; MOG, middle occipital gyrus.

nitive evaluations of perceptual discriminations may be best conceived of as an online monitor that integrates information over short time scales (Fleming and Dolan, 2012). Converging evidence from primate, electrophysiological, and functional imaging studies implicates the ACC in monitoring task performance (for review, see Ridderinkhof et al., 2004). For instance, activity in dACC increases in response to errors compared with correct responses (Ullsperger and von Cramon, 2004) and shows increased activation in trials characterized by high response conflict (e.g., Kerns et al., 2004). Recent evidence also suggests that the striatum, along with dACC and lateral PFC, contributes directly to monitoring and control over task performance (Balleine et al., 2007; Holroyd and Yeung, 2012). For instance, a recent study found that resting-state functional connectivity between the dACC and ventral striatum correlates with the percentage of times an individual "opts-out" of a perceptual decision under ambiguous circumstances (Jung et al., 2013). As recognizing ambiguity during decision-making depends on assessing confidence of what the correct action would be, the role of striatal regions in opting out of perceptual decisions supports a role of these regions in facilitating accurate metacognitive judgment, particularly when decision conflict is high. Overall, these results support the proposal by Fleming and Dolan (2012) that accurate metacognitive judgments of performance depend on the integrity with which lateral aPFC integrates performance-monitoring information coded in ACC.

By contrast, accurate metacognitive judgments of memory may be understood as a direct appraisal of memory contents or assessment of the strength of a memory trace (Nelson and $\mathrm{Na}$ rens, 1990). It is well known, based on both functional imaging and lesion studies, that the acquisition and long-term storage of information depend on structures in the medial temporal lobe (MTL) (Squire, 1992; Tulving, 2002). We found that a network of regions that included the parahippocampal gyrus showed a relative increase in connectivity with medial compared with lateral aPFC with increasing memorial metacognitive accuracy, providing support for a role of MTL structures in metacognitive assessment of memory. Furthermore, MTL memory structures are densely interconnected with both the central precuneus and the adjacent IPL/IPS (Cavanna and Trimble, 2006; Rushworth et al., 2006; Vincent et al., 2006; Margulies et al., 2009), suggesting that the reciprocal anatomical connections between these regions enable them to cooperate in cognitive processes related to memory. Indeed, our observation of the involvement of IPL in metacognitive assessment of memory is consistent with a role for this region either as an accumulator for the strength of evidence for or against a memory decision (Wagner et al., 2005) or as an output buffer that dynamically represents retrieved information (Baddeley, 2000; Vilberg and Rugg, 2008). More generally, the medial aPFC, the central precuneus, the inferior parietal lobule, as well as the MTL, are all implicated in the default mode network (Buckner et al., 2008; Andrews-Hanna et al., 2010), a system that is important in range of episodic simulations based on memory (Schacter et al., 2012) rather than perception (Smallwood et al., 2013). One question that naturally arises from our results, therefore, is what process, or processes, are common to metacognitive assessments of memory and other forms of memory-driven cognition, such as mind-wandering, prospection, and metalizing that this network is thought to support (for a meta-analysis, see Spreng et al., 2009).

To our knowledge, only one other study (McCurdy et al., 2013) has compared metacognitive ability for perceptual and memorial judgments within individuals. The present connectivity results and the voxel-based morphometry findings of McCurdy et al. (2013) converge in suggesting that individual differences in metacognitive ability for perception and memory relate to distinct features of brain architecture. Both studies suggest a primary role of lateral aPFC in metacognitive ability for perceptual discriminations and a role of the precuneus in metacognitive ability for memory. However, one difference between the present findings and those of McCurdy et al. (2013) is that, despite the dissociation at the neural level, McCurdy et al. (2013) found a positive correlation between behavioral scores for perceptual and memorial metacognitive ability. One possibility is that this discrepancy could be attributed to differences between the tasks. For example, our memory task required a longer retention interval than the task used by McCurdy et al. (2013). Additionally, the memory task used by McCurdy et al. (2013) was a two-alternative forced choice task, whereas the present study used a two-choice old/new discrimination task, and there are differences between these two types of memory tests in the recruitment of recollection (Cook et al., 2005). Although both studies differentiate metacognitive ability at the neural level, larger-scale behavioral studies 
manipulating factors, such as retention interval length, as well as the type and uncertainty of the type I discrimination, will be necessary before firm conclusions can be drawn regarding the behavioral stability of metacognitive capacity across domains.

In conclusion, our data demonstrate that accurate metacognitive assessments of perception and memory relate to the strength of functional coupling within distinct large-scale cortical networks involving the medial and lateral regions of aPFC. Although these findings call into question a strict homogeneity of metacognition, it is important to note that the individual differences approach used here identifies cross-sectional differences that underlie the capacity for accurate metacognitive assessment rather than an exhaustive account of the neural processes that contribute to metacognitive judgments in either domain. It remains possible, therefore, that domain-general attentional or cognitive processes could be involved in the evaluation of both memorial and perceptual judgments, a possibility that is supported by task-based comparisons of confidence judgments in memory and perception (Fleck et al., 2006). As it seems likely that in many situations information from perceptual and memorial sources is processed in tandem to maximize behavioral flexibility, a challenge for future research is to understand how these different sources of information can be integrated in order for an individual to navigate the complex, and often unpredictable, environment in which they exist.

\section{References}

Andrews-Hanna JR, Reidler JS, Sepulcre J, Poulin R, Buckner RL (2010) Functional-anatomic fractionation of the brain's default network. Neuron 65:550-562. CrossRef Medline

Avants BB, Tustison NJ, Song G, Cook PA, Klein A, Gee JC (2011) A reproducible evaluation of ANTs similarity metric performance in brain image registration. Neuroimage 54:2033-2044. CrossRef Medline

Bayen R, Piepenbrock R, Gulikers L (1995) The CELEX lexical database (version release 2)[CD-ROM]. Philadelphia: Linguistic Data Consortium, University of Pennsylvania.

Baddeley A (2000) The episodic buffer: a new component of working memory? Trends Cogn Sci 4:417-423. CrossRef Medline

Balleine BW, Delgado MR, Hikosaka O (2007) The role of the dorsal striatum in reward and decision-making. J Neurosci 27:8161-8165. CrossRef Medline

Behzadi Y, Restom K, Liau J, Liu TT (2007) A component based noise correction method (CompCor) for BOLD and perfusion based fMRI. Neuroimage 37:90-101. CrossRef Medline

Belyi BI (1987) Mental impairment in unilateral frontal tumours: role of the laterality of the lesion. Int J Neurosci 32:799-810. CrossRef Medline

Brainard DH (1997) The Psychophysics Toolbox. Spat Vis 10:433-436. CrossRef Medline

Buckner RL, Andrews-Hanna JR, Schacter DL (2008) The brain's default network: anatomy, function, and relevance to disease. Ann N Y Acad Sci 1124:1-38. CrossRef Medline

Cavanna AE, Trimble MR (2006) The precuneus: a review of its functional anatomy and behavioural correlates. Brain 129:564-583. CrossRef Medline

Christoff K, Gabrieli JDE (2000) The frontopolar cortex and human cognition: evidence for a rostrocaudal hierarchical organization within the human prefrontal cortex. Psychobiology 20:168-186.

Chua EF, Schacter DL, Rand-Giovannetti E, Sperling RA (2006) Understanding metamemory: neural correlates of the cognitive process and subjective level of confidence in recognition memory. Neuroimage 29:1150-1160. CrossRef Medline

Chua EF, Schacter DL, Sperling RA (2009) Neural correlates of metamemory: a comparison of feeling-of-knowing and retrospective confidence judgments. J Cogn Neurosci 21:1751-1765. CrossRef Medline

Chumbley J, Worsley K, Flandin G, Friston K (2010) Topological FDR for neuroimaging. Neuroimage 49:3057-3064. CrossRef Medline

Cook GI, Marsh RL, Hicks JL (2005) Revisiting the role of recollection in item versus forced-choice recognition memory. Psychon Bull Rev 12: 720-725. CrossRef Medline
Cox RW (2012) AFNI: what a long strange trip it's been. Neuroimage 62: 743-747. CrossRef Medline

Dale AM, Fischl B, Sereno MI (1999) Cortical surface-based analysis: I. Segmentation and surface reconstruction. Neuroimage 9:179-194. CrossRef Medline

Davidson PS, Anaki D, Ciaramelli E, Cohn M, Kim AS, Murphy KJ, Troyer AK, Moscovitch M, Levine B (2008) Does lateral parietal cortex support episodic memory? Evidence from focal lesion patients. Neuropsychologia 46:1743-1755. CrossRef Medline

Elman JA, Klostermann EC, Marian DE, Verstaen A, Shimamura AP (2012) Neural correlates of metacognitive monitoring during episodic and semantic retrieval. Cogn Affect Behav Neurosci 12:599-609. CrossRef Medline

Fischl B, Dale AM (2000) Measuring the thickness of the human cerebral cortex from magnetic resonance images. Proc Natl Acad Sci U S A 97: 11050-11055. CrossRef Medline

Fischl B, Sereno MI, Dale AM (1999a) Cortical surface-based analysis: II. Inflation, flattening, and a surface-based coordinate system. Neuroimage 9:195-207. CrossRef Medline

Fischl B, Sereno MI, Tootell RB, Dale AM (1999b) High-resolution intersubject averaging and a coordinate system for the cortical surface. Hum Brain Mapp 8:272-284. CrossRef Medline

Fischl B, Liu A, Dale AM (2001) Automated manifold surgery: constructing geometrically accurate and topologically correct models of the human cerebral cortex. IEEE Trans Med Imaging 20:70-80. CrossRef Medline

Fischl B, Salat DH, Busa E, Albert M, Dieterich M, Haselgrove C, van der Kouwe A, Killiany R, Kennedy D, Klaveness S, Montillo A, Makris N, Rosen B, Dale AM (2002) Whole brain segmentation: automated labeling of neuroanatomical structures in the human brain. Neuron 33:341355. CrossRef Medline

Fischl B, Salat DH, van der Kouwe AJ, Makris N, Ségonne F, Quinn BT, Dale AM (2004a) Sequence-independent segmentation of magnetic resonance images. Neuroimage 23 [Suppl 1]:S69-S84.

Fischl B, van der Kouwe A, Destrieux C, Halgren E, Ségonne F, Salat DH, Busa E, Seidman LJ, Goldstein J, Kennedy D, Caviness V, Makris N, Rosen B, Dale AM (2004b) Automatically parcellating the human cerebral cortex. Cereb Cortex 14:11-22. CrossRef Medline

Fleck MS, Daselaar SM, Dobbins IG, Cabeza R (2006) Role of prefrontal and anterior cingulate regions in decision-making processes shared by memory and nonmemory tasks. Cereb Cortex 16:1623-1630. CrossRef Medline

Fleming SM, Dolan RJ (2012) The neural basis of metacognitive ability. Philos Trans R Soc Lond B Biol Sci 367:1338-1349. CrossRef Medline

Fleming SM, Weil RS, Nagy Z, Dolan RJ, Rees G (2010) Relating introspective accuracy to individual differences in brain structure. Science 329: 1541-1543. CrossRef Medline

Fleming SM, Huijgen J, Dolan RJ (2012) Prefrontal contributions to metacognition in perceptual decision making. J Neurosci 32:6117-6125. CrossRef Medline

Fox MD, Snyder AZ, Vincent JL, Corbetta M, Van Essen DC, Raichle ME (2005) The human brain is intrinsically organized into dynamic, anticorrelated functional networks. Proc Natl Acad Sci U S A 102:9673-9678. CrossRef Medline

Galvin SJ, Podd JV, Drga V, Whitmore J (2003) Type 2 tasks in the theory of signal detectability: discrimination between correct and incorrect decisions. Psychon Bull Rev 10:843-876. CrossRef Medline

Gilbert SJ, Spengler S, Simons JS, Steele JD, Lawrie SM, Frith CD, Burgess PW (2006) Functional specialization within rostral prefrontal cortex (area 10): a meta-analysis. J Cogn Neurosci 18:932-948. CrossRef Medline

Gorgolewski K, Burns CD, Madison C, Clark D, Halchenko YO, Waskom ML, Ghosh SS (2011) Nipype: a flexible, lightweight and extensible neuroimaging data processing framework in python. Front Neuroinform 5:13. CrossRef Medline

Green DM, Swets JA (1966) Signal detection theory and psychophysics. New York: Wiley.

Greve DN, Fischl B (2009) Accurate and robust brain image alignment using boundary-based registration. Neuroimage 48:63-72. CrossRef Medline

Han X, Jovicich J, Salat D, van der Kouwe A, Quinn B, Czanner S, Busa E, Pacheco J, Albert M, Killiany R, Maguire P, Rosas D, Makris N, Dale A, Dickerson B, Fischl B (2006) Reliability of MRI-derived measurements of human cerebral cortical thickness: the effects of field strength, scanner upgrade and manufacturer. Neuroimage 32:180-194. CrossRef Medline 
Holroyd CB, Yeung N (2012) Motivation of extended behaviors by anterior cingulate cortex. Trends Cogn Sci 16:122-128. CrossRef Medline

Joseph R (1999) Frontal lobe psychopathology: mania, depression, confabulation, catatonia, perseveration, obsessive compulsions, and schizophrenia. Psychiatry 62:138-172. Medline

Jovicich J, Czanner S, Greve D, Haley E, van der Kouwe A, Gollub R, Kennedy D, Schmitt F, Brown G, Macfall J, Fischl B, Dale A (2006) Reliability in multi-site structural MRI studies: effects of gradient non-linearity correction on phantom and human data. Neuroimage 30:436-443. CrossRef Medline

Jung YC, Schulte T, Müller-Oehring EM, Hawkes W, Namkoong K, Pfefferbaum A, Sullivan EV (2013) Synchrony of anterior cingulate cortex and insular-striatal activation predicts ambiguity aversion in individuals with low impulsivity. Cereb Cortex Advance online publication. Retrieved January 24, 2013. doi: 10.1093/cercor/bht008. CrossRef Medline

Kerns JG, Cohen JD, MacDonald AW 3rd, Cho RY, Stenger VA, Carter CS (2004) Anterior cingulate conflict monitoring and adjustments in control. Science 303:1023-1026. CrossRef Medline

Kim H, Cabeza R (2007) Trusting our memories: dissociating the neural correlates of confidence in veridical versus illusory memories. J Neurosci 27:12190-12197. CrossRef Medline

Kleiner M, Brainard D, Pelli D, Ingling A, Murray R, Broussard C (2007) What's new in Psychtoolbox-3. Perception 36:1.1-16. CrossRef Medline

Kornbrot DE (2006) Signal detection theory, the approach of choice: model-based and distribution-free measures and evaluation. Percept Psychophys 68:393-414. CrossRef Medline

Levitt H (1971) Transformed up down methods in psychoacoustics. J Acoust Soc Am 49 [Suppl 2]:467.

Macmillan NA, Creelman CD (2004) Detection theory: a user's guide. New York: Psychology.

Maniscalco B, Lau H (2012) A signal detection theoretic approach for estimating metacognitive sensitivity from confidence ratings. Conscious Cogn 21:422-430. CrossRef Medline

Margulies DS, Vincent JL, Kelly C, Lohmann G, Uddin LQ, Biswal BB, Villringer A, Castellanos FX, Milham MP, Petrides M (2009) Precuneus shares intrinsic functional architecture in humans and monkeys. Proc Natl Acad Sci U S A 106:20069-20074. CrossRef Medline

McCurdy LY, Maniscalco B, Metcalfe J, Liu KY, de Lange FP, Lau H (2013) Anatomical coupling between distinct metacognitive systems for memory and visual perception. J Neurosci 33:1897-1906. CrossRef Medline

Metcalfe JE, Shimamura AP (1994) Metacognition: knowing approximately knowing. Boston: MIT.

Mickes L, Wixted JT, Wais PE (2007) A direct test of the unequal-variance signal detection model of recognition memory. Psychon Bull Rev 14:858865. CrossRef Medline

Modirrousta M, Fellows LK (2008) Medial prefrontal cortex plays a critical and selective role in 'feeling of knowing' meta-memory judgments. Neuropsychologia 46:2958-2965. CrossRef Medline

Moritz S, Gläscher J, Sommer T, Büchel C, Braus DF (2006) Neural correlates of memory confidence. Neuroimage 33:1188-1193. CrossRef Medline

Nelson TO, Narens L (1990) Metamemory: a theoretical framework and new findings. Psychol Learn Motivation 26:125-141. CrossRef

Öngür D, Ferry AT, Price JL (2003) Architectonic subdivision of the human orbital and medial prefrontal cortex. J Comp Neurol 460:425-449. CrossRef Medline

Pannu JK, Kaszniak AW (2005) Metamemory experiments in neurological populations: a review. Neuropsychol Rev 15:105-130. CrossRef Medline

Ramnani N, Owen AM (2004) Anterior prefrontal cortex: insights into function from anatomy and neuroimaging. Nat Rev Neurosci 5:184-194. CrossRef Medline

Ridderinkhof KR, Ullsperger M, Crone EA, Nieuwenhuis S (2004) The role of the medial frontal cortex in cognitive control. Sci Signal 306:443. CrossRef Medline

Roche A (2011) A four-dimensional registration algorithm with application to joint correction of motion and slice timing in fMRI. IEEE Trans Med Imaging 30:1546-1554. CrossRef Medline

Rounis E, Maniscalco B, Rothwell JC, Passingham RE, Lau H (2010) Thetaburst transcranial magnetic stimulation to the prefrontal cortex impairs metacognitive visual awareness. Cogn Neurosci 1:165-175. CrossRef

Rushworth MF, Behrens TE, Johansen-Berg H (2006) Connection pat- terns distinguish 3 regions of human parietal cortex. Cereb Cortex 16:1418-1430. CrossRef Medline

Schacter DL, Addis DR, Hassabis D, Martin VC, Spreng RN, Szpunar KK (2012) The future of memory: remembering, imagining, and the brain. Neuron 76:677-694. CrossRef Medline

Schmitz TW, Rowley HA, Kawahara TN, Johnson SC (2006) Neural correlates of self-evaluative accuracy after traumatic brain injury. Neuropsychologia 44:762-773. CrossRef Medline

Schnyer DM, Verfaellie M, Alexander MP, LaFleche G, Nicholls L, Kaszniak AW (2004) A role for right medial prefrontal cortex in accurate feelingof-knowing judgments: evidence from patients with lesions to frontal cortex. Neuropsychologia 42:957-966. CrossRef Medline

Schnyer DM, Nicholls L, Verfaellie M (2005) The role of VMPC in metamemorial judgments of content retrievability. J Cogn Neurosci 17:832-846. CrossRef Medline

Seeley WW, Menon V, Schatzberg AF, Keller J, Glover GH, Kenna H, Reiss AL, Greicius MD (2007) Dissociable intrinsic connectivity networks for salience processing and executive control. J Neurosci 27:2349-2356. CrossRef Medline

Ségonne F, Dale AM, Busa E, Glessner M, Salat D, Hahn HK, Fischl B (2004) A hybrid approach to the skull stripping problem in MRI. Neuroimage 22:1060-1075. CrossRef Medline

Simons JS, Davis SW, Gilbert SJ, Frith CD, Burgess PW (2006) Discriminating imagined from perceived information engages brain areas implicated in schizophrenia. Neuroimage 32:696-703. CrossRef Medline

Simons JS, Henson RN, Gilbert SJ, Fletcher PC (2008) Separable forms of reality monitoring supported by anterior prefrontal cortex. J Cogn Neurosci 20:447-457. CrossRef Medline

Simons JS, Peers PV, Mazuz YS, Berryhill ME, Olson IR (2010) Dissociation between memory accuracy and memory confidence following bilateral parietal lesions. Cereb Cortex 20:479-485. CrossRef Medline

Smallwood J, Tipper C, Brown K, Baird B, Engen H, Michaels JR, Grafton S, Schooler JW (2013) Escaping the here and now: evidence for a role of the default mode network in perceptually decoupled thought. Neuroimage 69:120-125. CrossRef Medline

Smith SM (1992) A new class of corner finder. In Proceedings of the British Machine Vision Conference, 22-24 September, Leeds, pp 139-148. London: Springer.

Song C, Kanai R, Fleming SM, Weil RS, Schwarzkopf DS, Rees G (2011) Relating inter-individual differences in metacognitive performance on different perceptual tasks. Conscious Cogn 20:1787-1792. CrossRef Medline

Spreng RN, Mar RA, Kim AS (2009) The common neural basis of autobiographical memory, prospection, navigation, theory of mind, and the default mode: a quantitative meta-analysis. J Cogn Neurosci 21:489-510. CrossRef Medline

Squire LR (1992) Memory and the hippocampus: a synthesis from findings with rats, monkeys, and humans. Psychol Rev 99:195-231. CrossRef Medline

Swets JA (1986) Form of empirical ROCs in discrimination and diagnostic tasks: implications for theory and measurement of performance. Psychol Bull 99:181-198. CrossRef Medline

Tulving E (2002) Episodic memory: from mind to brain. Annu Rev Psychol 53:1-25. CrossRef Medline

Ullsperger M, von Cramon DY (2004) Neuroimaging of performance monitoring: error detection and beyond. Cortex 40:593-604. CrossRef Medline

Vilberg KL, Rugg MD (2008) Memory retrieval and the parietal cortex: a review of evidence from a dual-process perspective. Neuropsychologia 46:1787-1799. CrossRef Medline

Vincent JL, Snyder AZ, Fox MD, Shannon BJ, Andrews JR, Raichle ME, Buckner RL (2006) Coherent spontaneous activity identifies a hippocampal-parietal memory network. J Neurophysiol 96:3517-3531. CrossRef Medline

Wagner AD, Shannon BJ, Kahn I, Buckner RL (2005) Parietal lobe contributions to episodic memory retrieval. Trends Cogn Sci 9:445-453. CrossRef Medline

Wheeler ME, Buckner RL (2004) Functional-anatomic correlates of remembering and knowing. Neuroimage 21:1337-1349. CrossRef Medline

Yokoyama O, Miura N, Watanabe J, Takemoto A, Uchida S, Sugiura M, Horie K, Sato S, Kawashima R, Nakamura K (2010) Right frontopolar cortex activity correlates with reliability of retrospective rating of confidence in short-term recognition memory performance. Neurosci Res 68:199-206. CrossRef Medline 